\title{
Behavioral Analysis of Second Order Sigma-Delta Modulator for Low frequency Applications
}

\author{
Susmita S. Samanta ${ }^{1}$, R.W. Jasutkar ${ }^{2}$ \\ ${ }^{1}$ (Department of Computer Science Engineering, G.H Raisoni College of Engineering,India) \\ ${ }^{2}$ (Department of Computer Science Engineering,G.H Raisoni College of Engineering,India)
}

\begin{abstract}
Switched capacitor (SC) based modulator is prone to various non-idealities; especially at the circuit designing stage where the integrator plays an important role and effects the overall performance of the sigma delta modulator. The non idealities take account of sampling jitter which includes the effect of switching circuitry, sampling noise. Opamp parameter include noise, finite $d c$ gain, finite band width, slew rate, saturation voltage. Each non idealities is modeled mathematically and simulation is carried out in the MATALAB SIMULINK ${ }^{\circledR}$ environment with the help of sigma delta toolbox. The simulation analysis of each model is carried out individually. At last overall performance of the modulator with all nonidealities is carried out compared with the ideal modulator and satisfactory results were found out for second order sigma delta modulator for signal bandwidth of $4 \mathrm{KHz}$.
\end{abstract}

Keywords: Sigma Delta modulator ( $\ 4$ ), Behavioral Analysis, Switch Capacitor (SC), Genetic Algorithm(GA).

\section{INTRODUCTION}

Sigma delta modulator are exploited in various applications like wireless communication devices, consumer electronics, biomedical applications. The oversampling property of the sigma delta modulator employ coarse quantization enclosed in one or more feedback loops. By sampling at a frequency much higher than the signal bandwidth it is possible for the feedback loops to shape the quantization noise so that most of the noise power is shifted out of the signal band [1]. This is illustrated in fig.1

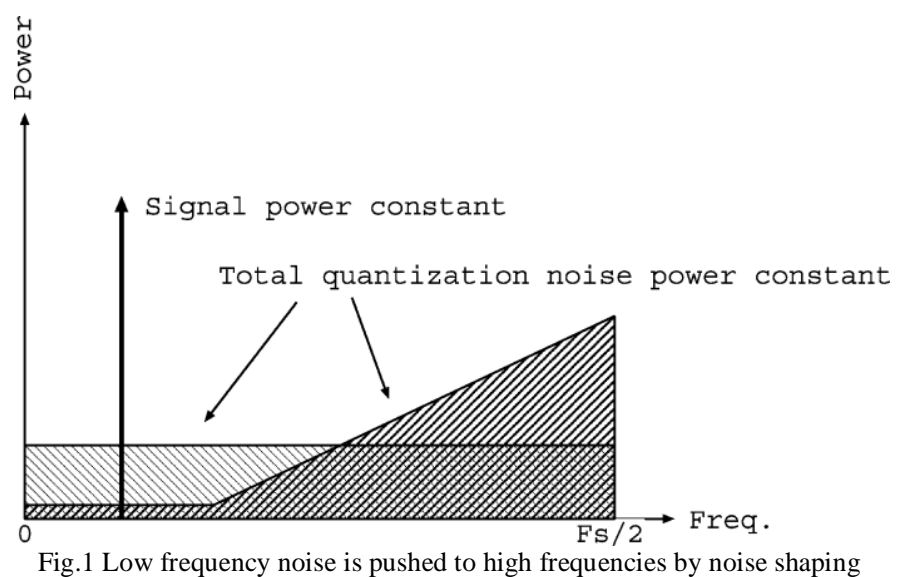

The out of band noise can then be attenuated with a digital filter. The degree to which the quantization noise can be attenuated depends on the order of the noise shaping and oversampling ratio. Sigma delta modulators are suitable for low frequency, high resolution applications, in keeping view of their linearity property, reduced requirement of the antialiasing filter, and its robustness. $\Sigma \Delta$ modulator allows high performance to achieve without effecting accuracy and speed with low sensitivity to analog component imperfections or component trimming.

$\Sigma \Delta$ modulators can be implemented either with continuous-time or with discrete-data techniques. The most popular approach is based on a discrete-data solution with switched capacitor (SC) implementation. As the technology is scaling down into a deep submicron level transistor sizing has become an issue. The fact that,SC $\Sigma \Delta$ modulators can be effectively realized in standard CMOS technology without any performance degradation. During the design phase of an SDM the noise-shaping transfer function is typically evaluated using a linear model. For a 1-bit quantizer, the noise transfer is highly non-linear and large differences between predicted and actual realized transfer can occur. The linear modeling is used to evaluate the performance of the sigma delta modulator through simulation [2]. Various criteria exits to evaluate the performance of the SMD. 
In practice, a significant problem in the design of the sigma delta modulators is the estimation of their performance, science they are mixed signal non linear circuits. Due to the nonlinearity of the modulator loop the optimization of the performance has to be carried out with behavioral time domain analysis. For high performance accurate simulation of number of non idealities and eventually, comparison of performance of different architecture are needed in order to choose the best possible solution. A large set of parameters need to optimize so as to achieve the desired signal to noise ratio (SNR). Therefore, in this paper we present a complete set of SIMULINK models, which allow us to perform exhaustive time-domain behavioral analysis of any $\Sigma \Delta$ modulator taking into account most of the non-idealities, such as sampling jitter, $k T / C$ noise and operational amplifier parameters (noise, finite dc gain, finite bandwidth, slew-rate (SR) and saturation voltages). The following sections describe in detail each of the models presented. Finally, simulation results, which demonstrate the validity of the models proposed, are provided. All the simulations were carried out on 2 nd-order $\mathrm{SC} \Sigma \Delta$ modulator architecture.

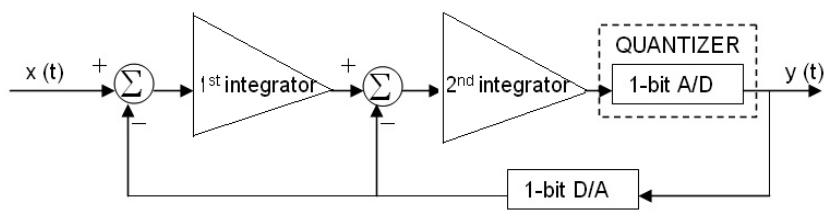

Fig. 2 Block diagram of $2^{\text {nd }}$ order $\Sigma \Delta$ modulator

II. NON-IDEALITIES IN SMD

The block diagram of second order sigma delta modulator is shown in Fig.2 . The modulator consist of input sampler, an integrator, a quantizer/comparator and feedback loop which consist of a digital to analog converter (DAC).Depending upon the number of the integrator the order of the modulator is decided. The integrator integrates the input signal over on each clock cycle, which operates a much higher frequency than the sampling frequency. This property of oversampling is being exploited in the sigma delta modulator. The integration of the pulse difference is linear over one clock cycle. The output of the integrator is then fed to the quantizer. The quantizer then digitized the input signal. The feedback path shifts the logic level of the input, so that it matches the logic level of the input.

The schematic of a first-order SC $\Sigma \Delta$ modulator is shown in Fig.3. The main nonidealities appearing in the SC circuitry, which should be considered for accurate modeling, are as follows:

- Clock jitter

- Switch thermal noise

- Operational amplifier noise

- Opamp DC gain

- Opamp Bandwidth(BW)

- Opamp slew rate(SR)

They will be thoroughly discussed in the following sections. The simulation environment will be done on the output samples in the time domain. The nonidealities mentioned above produce a deviation of the output samples from their ideal values.

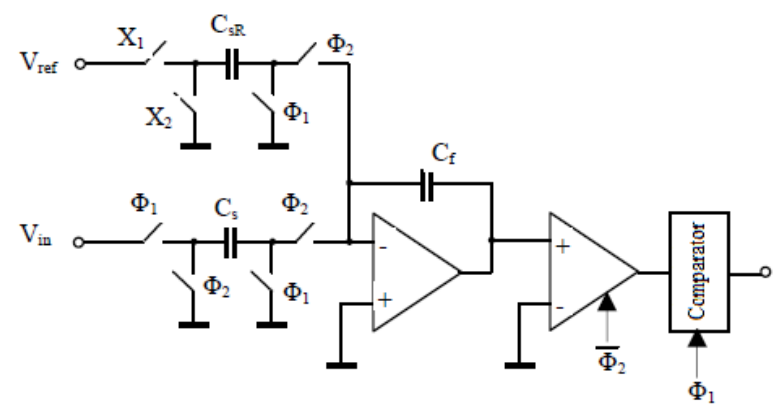

Fig. 3 Schematic of first order $\Sigma \Delta$ modulator

\section{CLOCK JITTER}

The clock jitter is sometimes referred as sampling jitter as it is determined by computing the effects on sampling the input signal. The jitter noise induced by the clock of is independent of the system architecture [4].The effect of sampling jitter on SC Sigma delta modulator can be calculated easily, since the operation of the 
SC depends on complete switching of the charge during each of the clock phase [5]. In fact, once the analog signal has been sampled, the SC circuit is a sampled data system where variations of the clock period have no direct effect on the circuit performance. Therefore, the effect of clock jitter on an SC circuit is completely described by computing its effect on the sampling of the input signal.

The clock jitter results in a non-uniform sampling time sequence and thus increases the total error power in the quantizer output. The magnitude of this error is a function of the statistical properties of the jitter and the input signal to the system. When the input is a sinusoidal, the error introduced by jitter can be modeled by

$$
x(t+\delta)-x(t) \approx 2 \pi f_{\text {in }} \delta A \cos \left(2 \pi f_{\text {in }} t\right)=\frac{\delta d x(t)}{d t}
$$

The input sinusoidal signal is $x(t), A$ is the amplitude of the signal. Frequency $f_{\text {in }}$ is sampled at an instant which is in error by an amount $\delta$ is given Eqn. (1). The input signal $x(t)$ and its derivative $(d u / d t)$ are continuous-time signals. They are sampled with sampling period $T S$ by a zero order hold. Here, we assumed that the sampling uncertainty $\delta$ is a Gaussian random process $n(t)$ with standard deviation $\Delta \tau$. The signal $n(t)$ is implemented starting from a sequence of random numbers with Gaussian distribution, zero mean, and unity standard deviation. Whether oversampling is helpful in reducing the error introduced by the jitter depends on the nature of the jitter. Since we assume the jitter white, the resultant error has uniform power-spectral density (PSD) from 0 to $f s / 2$. This effect can be simulated with SIMULINK ${ }^{\circledR}$ by using the model shown in Fig.4, which implements Eqn. (1).

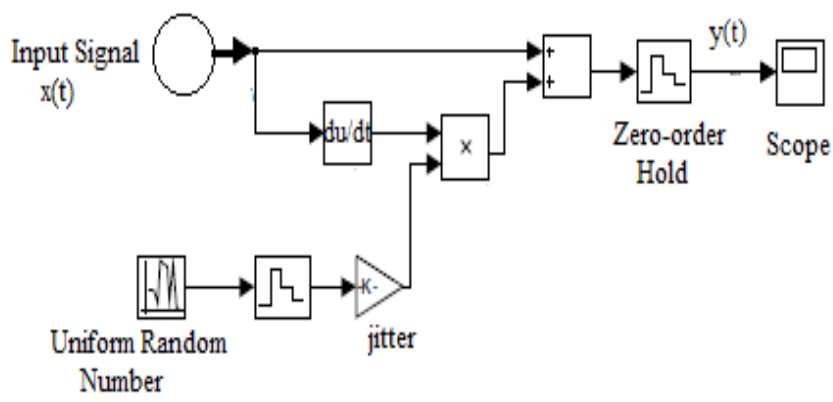

Fig.4 SIMULINK model for random clock jitter

\section{NOISE ON INTEGRATOR}

The most dominant noise sources affecting the operation of a switch capacitor of SMD are the resistance of the switches in the on state and the amplifier [6]. The total noise power of the circuit is the sum of the theoretical loop quantization noise power, the switch noise power and the op-amp noise power. These effects can be simulated with SIMULINK using "noisy" integrator model shown in Fig. 5. The variable $a=$ $C_{s} / C_{r}$ represents the integrator coefficient. The noise source like switches thermal noise and operational amplifier noise and its relevant model is described in the following sub-sections.

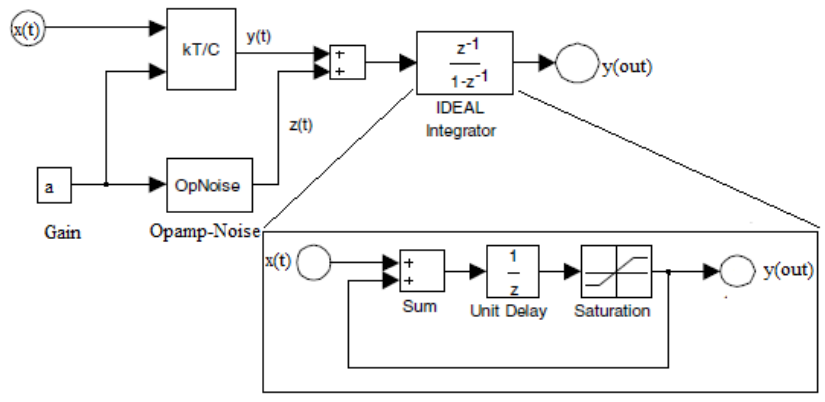

Fig. 5 "Noisy" Integrator Model

\section{A. Switches Thermal Noise}

The thermal noise associated to the sampling switches and the intrinsic noise of the operational amplifiers effects the operation of the modulator. According to Nyquist theorem the spectral density of noise at the terminals of a dipole passive depends only on the temperature and the real part of impedance of the dipole. 
In a switched capacitor integrator switches operate in ohmic region, the noise power at their terminals is equal to:

$$
E_{s f f}^{2}=\gamma(f) \Delta f=4 K T R \Delta f
$$

The noise due to switch on phases I and P is given by the Eqn. (3) and (4) respectively:

$$
\begin{aligned}
& V_{t h P}^{2}=\frac{K T}{C_{s}}+\frac{C_{r . K T}^{2}}{C_{s}^{2} \cdot C_{r}} \\
& V_{t h I}^{2}=\frac{K T}{C_{s}}+\frac{K T}{C_{r}}
\end{aligned}
$$

$K$ is the Boltzmann constant and $T$ the absolute temperature in Kelvin. The equations (3) and (4) show that if we wishes to reduce the thermal noise power, then we must increase the

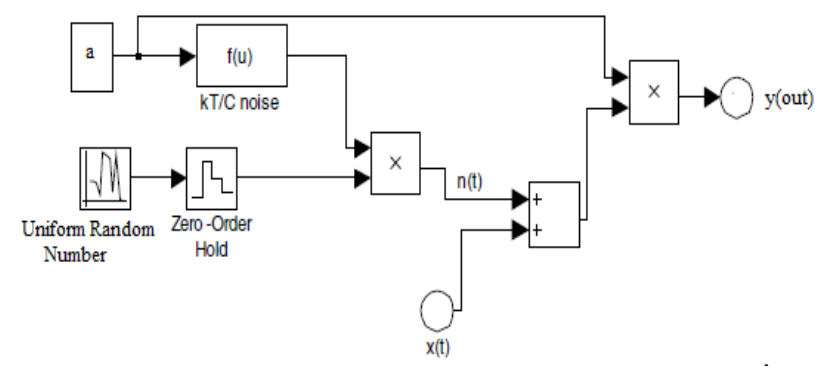

Fig. 6 Model of switches thermal noise

value of the sampling capacity $C s$ [7]. Thus the total noise can be evaluated by the following equation:

$$
e_{T}^{2}=\frac{K T}{C_{s}}
$$

The switch thermal noise voltage $e T$ (usually called $k T / C$ noise) is then superimposed to the input voltage $x(t)$ leading to:

$$
y(\text { out })=\left[x(t)+e_{t}(t)\right] b=\left(x(t)+\sqrt{\frac{K T}{C_{s}}} n(t)\right) b
$$

where $n(t)$ denotes a Gaussian random process with unity standard deviation, while $b$ is the integrator gain Eqn. 6 is implemented by the model shown in Fig. 6 .

\section{B. Operational Amplifier Noise}

The sources of noise present in an amplifier are generally two reasons for the thermal noise and noise in $1 / f$. For a MOS transistor in saturation, the voltage generator noise equivalent to the two sources is given by [7]:

$$
S_{E n} \approx 2 \frac{2}{3} K T \cdot g_{m}{ }^{-1}+\left(\frac{K_{f}}{C_{o x} W L}\right) \cdot \frac{1}{f}
$$

Fig. 7 shows the model used to simulate the effect of the operational amplifier noise.

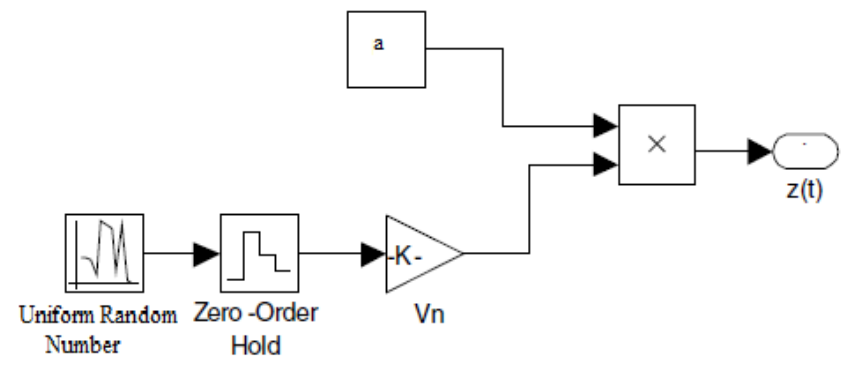

Fig.7 SIMULINK model of Operational Amplifier Noise

Here, $V n$ represents the total $r m s$ noise voltage referred to the op-amp input. Flicker (1/f) noise, wide-band thermal noise and the dc offset contribute to this value. The total op-amp noise power $(V n)^{2}$ can be evaluated, through circuit simulation, on the circuit of Fig. 2 during phase $\Phi 2$, by adding the noise contributions of all the devices referred to the op-amp input and integrating the resulting value over the whole frequency spectrum. 


\section{NONIDEALITIES OF INTEGRATOR}

Analog circuit implementations of the integrator deviate from this ideal behavior due to several nonideal effects. One of the major causes of performance degradation in SC $\Sigma \Delta$ modulators, indeed, is due to incomplete transfer of charge in the SC integrators. This non-ideal effect is a consequence of the op-amp nonidealities, namely finite gain and BW, slew rate (SR) and saturation voltages. These will be considered separately in the following subsections. Fig. 8 shows the model of the real integrator including all the nonidealities.

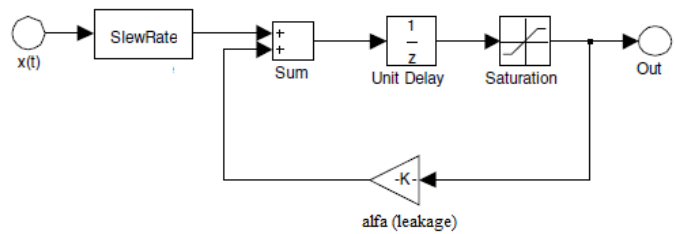

\section{A. DC Gain}

Fig.8 Real Integrator model

The DC gain of the ideal integrator is infinite. In practice, the gain of the operational amplifier open loop $A_{0}$ is finite. This is reflected by the fact that a fraction of the previous. Sample out of the integrator is added to the sample input [8]. The model of a real integrator with an integrator delay is real considering the saturation op-amp, the gain over the finite bandwidth and slew-rate. The $\mathrm{Z}$ transfer function of a perfect integrator is given by:

$$
H(z)=\frac{Z^{-1}}{1-Z^{-1}}
$$

The transfer function of the real integrator becomes:

$$
H(z)=\beta \frac{Z^{-1}}{1-\alpha Z^{-1}}
$$

where $\alpha$ and $\beta$ are the integrator's gain and leakage, respectively [9].

$$
\propto=\frac{A_{0}-1}{A_{0}}
$$

The limited gain at low frequencies increases the in-band noise.

\section{B. Bandwidth and $S R$}

The distortion limits the power effectively used by the system and its bandwidth. There are various reasons why a signal distorts. Regarding the harmonic distortion, it is mainly due to two factors of nonlinearity and slew-rate of the amplifiers

\section{a) Nonlinearity of the amplifiers}

Theoretically its transfer function is:

$$
V_{s}=A \cdot V_{e}
$$

$A$ is the amplification factor whose transfer function is approximated given by (12) [10].

$$
A \cong\left\{1+\propto_{1}\left|V_{0}\right|+\propto_{2}\left|V_{0}\right|^{2}+\propto_{3}\left|V_{0}\right|^{3}+\ldots\right\}
$$

Where $\left(\propto_{1}, \propto_{2}, \propto_{3}, \ldots\right)$ are the amplification factors parasites. Thus, for a pure sinusoidal signal of frequency $f$ in the input of the amplifier, we find the output of the amplifier, amplify the output signal with other parasitic elements and proportional to the frequency $f_{r}$, in this case we say that there is harmonic distortion, because this spectrum of frequencies $2 f, 3 f$, etc... . The total harmonic distortion is the ratio of the sum of squared amplitudes of these signals on the amplitude of the fundamental.

\section{b) Amplifier Slew Rate}

For given constant amplitude, slew-rate characterizes the limit of the amplifier frequency (maximal speed). When a signal is changing more slowly than the maximal speed, the amplifier follows and reproduces faithfully the signal. But when the signal frequency increases (for constant amplitude), the amplifier distorts the output signal. In this case, in addition to the original signal, there are additional frequencies (harmonics). The 
augmentation of the input signal frequency creates difficulty to the amplifier to restore the signal faithfully. For the amplifier responds linearly, it generally we define a maximum frequency above which the amplifier distorts the output signal. For a sinusoidal signal of amplitude $A$ pulsation $\omega$, this frequency is defined by

For a converter it is:

$$
G B W=2 \pi A f \leq S R \Rightarrow f=\frac{S R}{2 \pi A}
$$

$$
f=\frac{1}{2 \pi A 2^{n} \tau}
$$

$T$ is the settling time, $\tau=1 / 2 \pi G B W$ and $n$ the resolution of the converter. In the case of a switched-capacitor integrator (the main constituent of a $\Sigma \Delta$ modulator) the maximum speed cause an error "settling error" on the output voltage of the integrator. Indeed, the finite width of the band and "slew-rate" of the amplifier are related and may occur in the switched-capacitor circuit transient response non-ideal operation, "slew-rate" producing each clock tick an incomplete charge transfer at the end of the integration period. The effect of the finite bandwidth and the slew-rate are related to each other and may be interpreted as a non-linear gain.

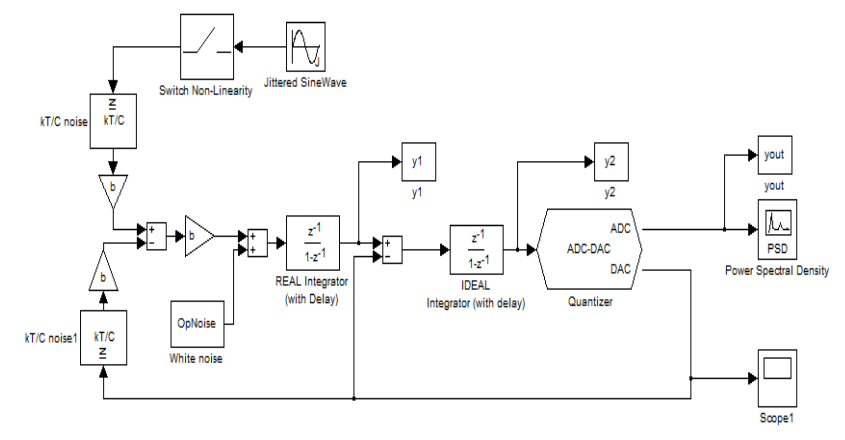

Fig. 9 SIMULINK model of second-order $\Sigma \Delta$ modulator

\section{Simulation Results}

To validate the proposed model designed for various non-idealities, we perform various simulations on the SIMULINK model on the model shown in Fig.9. A minimum SNDR of 90dB is required for the low frequency biomedical applications. The simulation parameters used for simulation are summarized in Table I. Table II compares the total SNDR and the corresponding effective number of bits (ENOB) which is nothing but the maximum number of resolution can be obtained from the architecture.

TABLE I

SIMULATION PARAMETERS

\begin{tabular}{c|c}
\hline Parameter & Value \\
\hline Signal Bandwidth & $\mathrm{BW}=4 \mathrm{Khz}$ \\
Sampling frequency & $\mathrm{F}_{\mathrm{s}}=1.008 \mathrm{MHz}$ \\
Oversampling Ratio & $\mathrm{R}=126$ \\
Sampling number & $\mathrm{N}=16384$ \\
Integrator Gain & $\mathrm{b}_{1}=\mathrm{b}_{2}=0.5$ \\
\hline
\end{tabular}

TABLE II

\begin{tabular}{|c|c|c|}
\hline $\begin{array}{c}2^{\text {nd }} \text { order } \Sigma \Delta \\
\text { Modulator } \\
\text { Parameter }\end{array}$ & $\begin{array}{c}\text { SNDR } \\
{[d B]}\end{array}$ & $\begin{array}{c}\text { ENOB } \\
\text { [bits] }\end{array}$ \\
\hline Ideal & 101.5 & 16.56 \\
\hline $\begin{array}{l}\text { Sampling jitter } \\
(\Delta \tau=6 \mathrm{~ns})\end{array}$ & 90.7 & 14.77 \\
\hline $\begin{array}{c}\text { Switches }(\mathrm{kT} / \mathrm{C}) \\
\text { noise }\left(\mathrm{C}_{\mathrm{s}}=6 \mathrm{pF}\right)\end{array}$ & 89.5 & 14.58 \\
\hline $\begin{array}{l}\text { Finite bandwidth } \\
(\mathrm{GBW}=8.6 \mathrm{MHz})\end{array}$ & 92.4 & 15.05 \\
\hline $\begin{array}{c}\text { Slew Rate } \\
(\mathrm{SR}=16 \mathrm{~V} / \mu \mathrm{s})\end{array}$ & 90.4 & 14.72 \\
\hline $\begin{array}{l}\text { Saturation Voltages } \\
\left(V_{\max }= \pm 5 \mathrm{~V}\right)\end{array}$ & 89.4 & 14.56 \\
\hline
\end{tabular}

SIMULATION RESULTS 


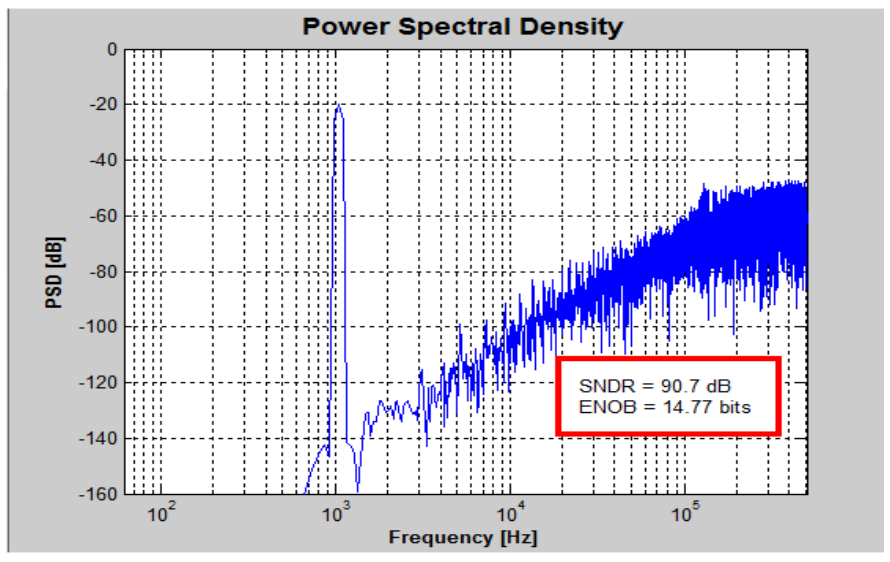

(a)

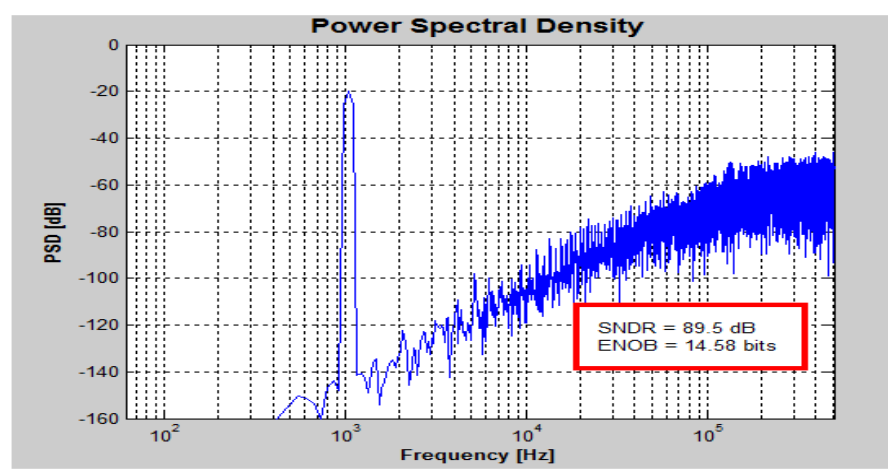

(b)

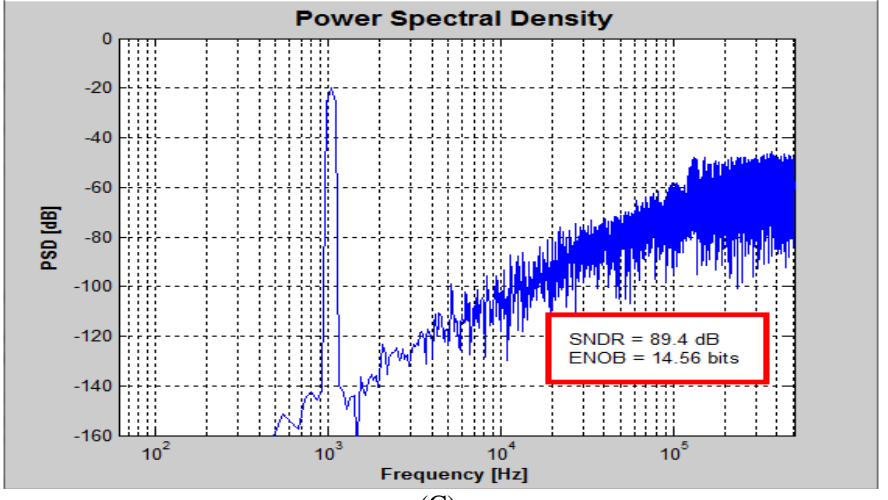

(C)

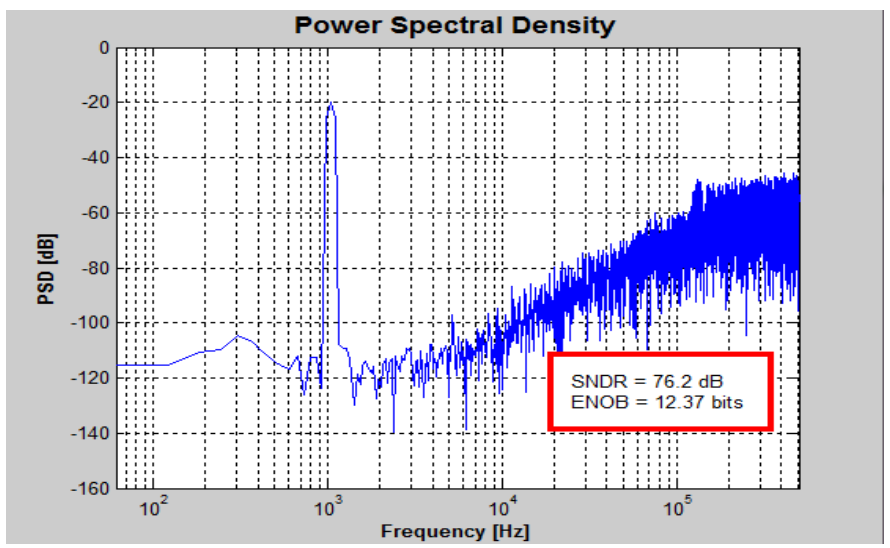

(d) 


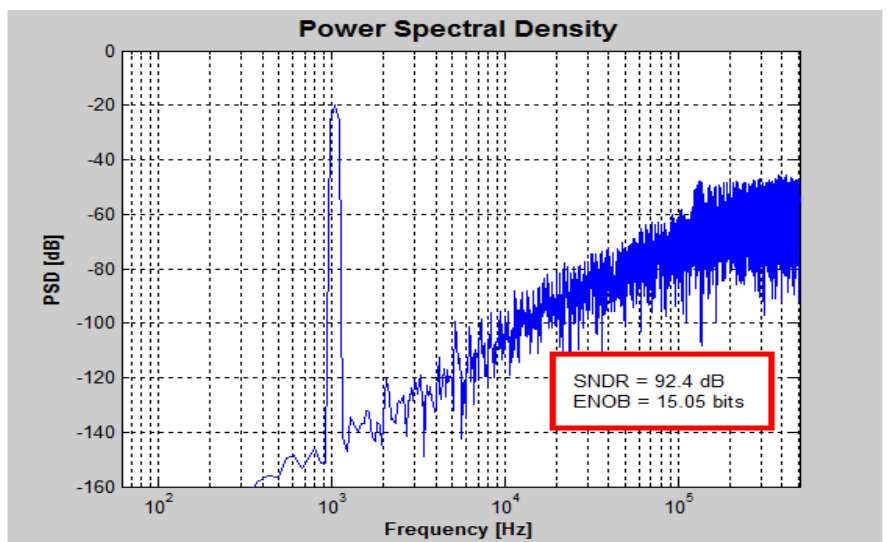

(e)

Fig. 10. PSD of (a) Sampling jitter $\Delta \tau=6 \mathrm{~ns}$ (b) Switches $(\mathrm{kT} / \mathrm{C})$ noise $\mathrm{C}_{\mathrm{s}}=6 \mathrm{pF}$ (c) GBW $=8.6 \mathrm{MHz}(\mathrm{d}) \mathrm{Slew}$ Rate $\mathrm{SR}=16 \mathrm{~V} / \mu \mathrm{s}$ (e) Saturation Voltage $V_{\max }= \pm 5 \mathrm{~V}$ )

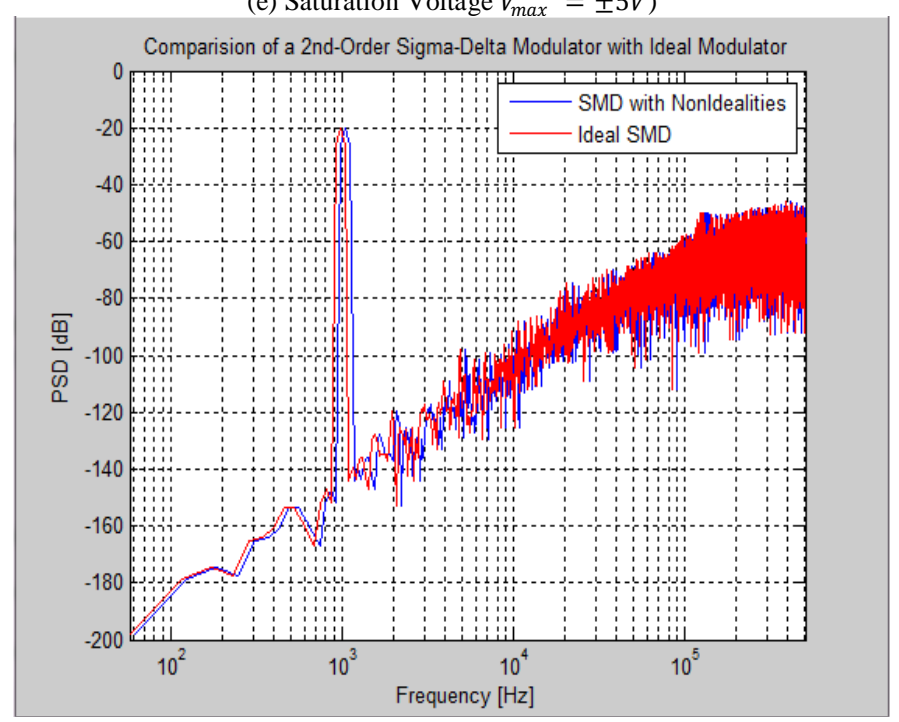

Fig. 11.Comparison plot of the Ideal SMD and SMD with Non Idealities

\section{CONCLUSION}

In this paper a modeling of second order sigma delta modulator is studied with various nonidealities of the modulator like operational amplifier noise, thermal noise, finite DC gain and comparison of ideal and non ideal SMD has also been done. The experimental results show that, SMD which is presented here have similarity with the ideal modulator. The performance of the second order $\Sigma \Delta$ modulator can be further being improved with the help of evolutionary algorithms like genetic algorithm (GA). Future work can be done with modeling of the SMD with GA.

\section{REFERENCES}

[1] Dragi.a Milovanović , Milan Savić, Miljan Nikolić, "Second-Order Sigma-Delta Modulator in Standard CMOS Technology", SERBIAN JOURNAL OF ELECTRICAL ENGINEERING Vol. 1, No. 3, November 2004, 37 - 44

[2] E. Janssen, A. van Roermund, "Look-Ahead Based Sigma-Delta Modulation", Analog Circuits and Signal Processing, DOI 10.1007/978-94-007-1387-1_2, ( ) Springer Science+Business Media B.V. 2011

[3] Shashant Jaykar, Prachi Palsodkar, Pravin Dakhole, "Modeling of Sigma-Delta Modulator Non-Idealities in MATLAB/SIMULINK", 2011 International Conference on Communication Systems and NetworkTechnologies, 978-0-7695-4437-3/11 \$26.00 (C) 2011 IEEE DOI 10.1109/CSNT.2011.112

[4] E. Farshidi, S. M. Sayedi, "A Second-order Low Power Current- Mode Continuous-Time Sigma-Delta Modulator," ASICON'07, Proceeding of the 7th IEEE International Conference on ASIC, Guilin, China, pp.293- 296, Oct. 2007

[5] E. Boser and B. A. Wooley, "The Design of Sigma-Delta Modulation Analog-to-Digital Converters", IEEE J. Solid- State Circ., vol. 23, pp. 1298-1308, Dec. 1988

[6] S. Brigati, F. Francesconi, P. Malcovati, D. Tonietto, A. Baschirotto and F. Maloberti, "MODELING SIGMA-DELTA MODULATOR NONIDEALITIES IN SIMULINK®,” IEEE International Symposium, pp 384 - 387 vol.2, 1999.

[7] Abdelghani Dendouga , Nour-Eddine Bouguechal, Souhil Kouda and Samir Barra, "Modeling of a Second Order Non-Ideal SigmaDelta Modulator", International Journal of Electrical and Compute Engineering 5:6 2010

[8] G. Temes, "Finite amplifier gain and bandwidth effects in switched capacitor filters," IEEE J. Solid-State Circuits, vol. 15, pp. 358361, June1980.

[9] H. Zare-Hoseini and I. Kale, "On the effects of finite and nonlinear DCgain on the switched-capacitor delta-sigma modulators," in Proc. IEEE Int. Symp. Circuits Systems, May 2005, pp. 2547-2550. 
[10] F. Medeiro et al., "Modeling opamp-induced harmonic distortion for switched-capacitor SD modulator design," in IEEE Int. Symp. Circuits Systems, vol. 5, May-Jun. 1994, pp. 445-448.

[11] Ms.R.W.Jasutkar, Dr.P.R.Baja,j Dr.A.Y.Deshmukh, "GA Based Low Power Sigma Delta Modulator for Biomedical Applications", 978-1- 4244-9477-4/11/\$26.00 @2011 IEEE

[12] Youngcheol Chae and Gunhee Han "A Low Power Sigma Delta Modulator Using Class-C Inverter" in 2007 Symposium on VLSI circuits Digest of Technical Papers.

[13] Shanthi Pavan, and Prabu Sankar "Power Reduction in Continuous-Time Delta-Sigma Modulators Using the Assisted Opamp Technique" in IEEE Journal Of Solid-State Circuits, Vol. 45, No. 7, July 2010.

[14] Arnold R. Feldman, Bernhard E. Boser, and Paul R. Gray, Fellow, "A 13-Bit, 1.4-MS/s Sigma-Delta Modulator for RF Baseband Channel Applications" in IEEE Journal Of Solid-State Circuits, Vol. 33, No. 10, October 1998.

[15] M.Loulou, D.Dallet and P.Marchegay "A 3.3V Switched Current Second Order Sigma Delta Modulator for Audio Applications" in IEEE 2000.

[16] Ebrahim Farshidi, Navid Alaei-Sheini “A Micropower Current Mode Sigma Delta Modulator for Biomedical Applications" in IEEE 2009.

[17] Chen Yueyang, Zhong Shun'an, Dang Hua "Design of A low-power consumption and high-performance sigma-delta Modulator" in 2009World Congress on Computer Science and Information Engineering 978-0-7695-3507-4/08 \$25.00 @ 2008 IEEE.

[18] Guo-Ming Sung, Chih-Ping Yu, Dong-An Yao "A Comparison of Second-Order Sigma-Delta Modulator Between SwitchedCapacitor and Switched-Current Techniques" in 978-1-4244-2342-2/08/\$25.00 @2008 IEEE. 